\title{
Anaerobic bacteria cultured from cystic fibrosis airways correlate to milder disease: a multisite study
}

\author{
Marianne S. Muhlebach ${ }^{1,2}$, Joseph E. Hatch ${ }^{2,3}$, Gisli G. Einarsson ${ }^{4,5}$, \\ Stef J. McGrath ${ }^{4}$, Deirdre F. Gilipin ${ }^{4}$, Gillian Lavelle ${ }^{6}$, Bojana Mirkovic $\mathbb{1}^{6}$, \\ Michelle A. Murray ${ }^{6}$, Paul McNally (107, , Nathan Gotman?, \\ Sonia Davis Thomas ${ }^{9,10}$, Matthew C. Wolfgang ${ }^{2,11}$, Peter H. Gilligan ${ }^{12,13}$, \\ Noel G. McElvaney ${ }^{6}$, J. Stuart Elborn ${ }^{5,14}$, Richard C. Boucher ${ }^{2}$ and \\ Michael M. Tunney ${ }^{4,5}$
}

@ERSpublications

Anaerobic bacteria are cultured across all ages, occur as communities and correlate with milder CF disease in adults http://ow.ly/7wQ430khMmE

Cite this article as: Muhlebach MS, Hatch JE, Einarsson GG, et al. Anaerobic bacteria cultured from cystic fibrosis airways correlate to milder disease: a multisite study. Eur Respir J 2018; 52: 1800242 [https://doi. org/10.1183/13993003.00242-2018].

ABSTRACT Anaerobic and aerobic bacteria were quantitated in respiratory samples across three cystic fibrosis (CF) centres using extended culture methods. Subjects aged 1-69 years who were clinically stable provided sputum $(n=200)$ or bronchoalveolar lavage $(n=55) .18$ anaerobic and 39 aerobic genera were cultured from $59 \%$ and $95 \%$ of samples, respectively; 16 out of 57 genera had a $\geqslant 5 \%$ prevalence across centres.

Analyses of microbial communities using co-occurrence networks in sputum samples showed groupings of oral, including anaerobic, bacteria, whereas typical CF pathogens formed distinct entities. Pseudomonas was associated with worse nutrition and F508del genotype, whereas anaerobe prevalence was positively associated with pancreatic sufficiency, better nutrition and better lung function. A higher total anaerobe/ total aerobe CFU ratio was associated with pancreatic sufficiency and better nutrition. Subjects grouped by factor analysis who had relative dominance of anaerobes over aerobes had milder disease compared with a Pseudomonas-dominated group with similar proportions of subjects that were homozygous for F508del.

In summary, anaerobic bacteria occurred at an early age. In sputum-producing subjects anaerobic bacteria were associated with milder disease, suggesting that targeted eradication of anaerobes may not be warranted in sputum-producing CF subjects. 
Affiliations: ${ }^{1}$ Dept of Pediatrics, University of North Carolina at Chapel Hill, Chapel Hill, NC, USA. ${ }^{2}$ Marsico Lung Institute, University of North Carolina at Chapel Hill, Chapel Hill, NC, USA. ${ }^{3}$ Dept of Pediatrics, Indiana University, Indianapolis, IN, USA. "Halo Research Group, School of Pharmacy, Queen's University Belfast, Belfast, UK. ${ }^{5}$ Halo Research Group, Centre for Experimental Medicine, School of Medicine, Dentistry and Biomedical Sciences, Queen's University Belfast, Belfast, UK. ${ }^{6}$ Respiratory Research Division, Dept of Medicine, Royal College of Surgeons in Ireland, Education and Research Centre, Beaumont Hospital, Dublin, Ireland. 'Dept of Paediatrics, Royal College of Surgeons in Ireland, Our Lady's Children's Hospital Crumlin, Dublin, Ireland. ${ }^{8} \mathrm{CF}$ Research Group, National Children's Research Centre, Our Lady's Children's Hospital Crumlin, Dublin, Ireland. ${ }^{9}$ Dept of Biostatistics, University of North Carolina at Chapel Hill, Chapel Hill, NC USA. ${ }^{10} \mathrm{RTI}$ International, Research Triangle Park, NC, USA. ${ }^{11}$ Dept of Microbiology, University of North Carolina at Chapel Hill, Chapel Hill, NC, USA. ${ }^{12}$ Pathology and Laboratory Medicine, University of North Carolina School of Medicine, Chapel Hill, NC, USA. ${ }^{13}$ Clinical Microbiology-Immunology Laboratories, UNC Health Care, Chapel Hill, NC, USA. ${ }^{14}$ Imperial College and Royal Brompton Hospital, London, UK.

Correspondence: Marianne S. Muhlebach, Dept of Pediatrics, University of North Carolina at Chapel Hill, 450 MacNider, Campus Box 7217, 333 South Columbia Street, Chapel Hill, NC 27599, USA.

E-mail: Marianne_Muhlebachamed.unc.edu

\section{Introduction}

Antibiotic therapies for cystic fibrosis (CF) respiratory infections are targeted at specific bacteria, typically Staphylococcus aureus, Pseudomonas aeruginosa and other Gram-negative species. However, both culture-dependent and culture-independent molecular methods have redefined CF respiratory infections as polymicrobial, with a diverse lower airways bacterial community [1]. Anaerobic conditions have been described in CF lower airways [2,3]. Importantly, obligate and facultative anaerobic bacteria, typically associated with the oral cavity, have been cultured in abundance from CF respiratory samples $[4,5]$. Indeed, extended anaerobic culture methods identified anaerobic bacteria in high numbers in $>60 \%$ of $\mathrm{CF}$ sputum samples from adults [6].

The role of anaerobic bacteria in CF lower airways disease is not clearly understood. Studies have identified anaerobic bacteria from direct lung samples, suggesting their presence does not simply reflect salivary contamination during sampling [7]. In vitro studies have demonstrated that anaerobes release virulence factors, including proteases and pro-inflammatory short-chain fatty acids $[8,9]$, suggesting that anaerobes may be pathogenic in the CF lung. In contrast, in vivo molecular studies indicate that greater anaerobe diversity is associated with milder disease $[10,11]$. Molecular methods highlight the breadth of bacteria, and novel preparation methods and metagenomics address a shortcoming of conventional next-generation sequencing, i.e. the amplification of sequences of nonviable bacteria [12-14]. Bacterial abundance can be measured by quantitative PCR; however, 16S rRNA copy numbers per genome vary between genera and quantitation of different genera in multispecies infections remains imprecise $[15,16]$. Culture allows enumeration of the different bacterial genera in a polymicrobial infection, demonstrates viability and growth in lower airways, and enables subsequent in vitro studies $[17,18]$. Culturing anaerobic and bacteria not typically considered as pathogenic is also relevant to clinical care, i.e. would knowledge about abundance of these bacteria be useful for treatment?

Here, we assessed the association of strict anaerobes with disease severity in CF across all age ranges at three CF centres with different genetic and geographic backgrounds and medications. Although "CF pathogens" can grow under anaerobic conditions, this likely reflects adaptation to the microenvironment and not distinct genera/infections. We used extended culture methods attempting to investigate the role of anaerobes in CF lung disease. We focused on strict anaerobic bacteria to match genera reported in molecular studies, and to ascertain the load and viability of these anaerobes in lower airways in relation to typical CF pathogens. Three key questions were investigated. 1) Does the prevalence of anaerobes versus aerobes in subjects with $\mathrm{CF}$ change as a function of age and site/climate? 2) Are there associations between prevalence or quantity of anaerobes and genotype, disease severity and medications? 3) Are community structures established by anaerobes and do they differ across ages, disease severity or associations with aerobes?

\section{Methods}

\section{Subjects and sample collection}

People diagnosed with CF attending CF centres in Belfast in the UK (Adult and Paediatric CF Clinics, Belfast Health and Social Care Trust), University of North Carolina (UNC) at Chapel Hill, NC in the USA (UNC Hospital Pediatric and Adult Clinics) and Dublin in Ireland (Beaumont Hospital and Our Lady's Children's Hospital, Crumlin) were prospectively enrolled into this study between July 2010 and November 2013. Subjects who had undergone transplant, were taking CF transmembrane conductance regulator potentiators/modulators or enrolled in interventional therapeutic trials were excluded. Ethical 
approval for the study was obtained at each institution, and informed consent/assent was obtained from all adults, parents and paediatric subjects.

Either bronchoalveolar lavage (BAL) or sputum (if able to expectorate) and concomitant clinical information were collected when subjects were clinically stable, defined as not having received antibiotics beyond chronic maintenance therapy and $\geqslant 6$ weeks post-completion of intravenous antibiotic therapy. Bronchoscopy was performed at UNC if a child had a procedure requiring anaesthesia and for routine surveillance at Dublin. At both sites, bronchoscopy was performed utilising a laryngeal mask airway or endotracheal tube.

\section{Clinical data}

Demographics, anthropometrics, lung function, medication history and disease complications were entered into a centralised database. Genotype was categorised by number of F508del alleles. Disease severity was assessed by nutritional status, pancreatic status and lung function (forced expiratory volume in $1 \mathrm{~s}$ (FEV1) \% pred; see supplementary material) if subjects were able to reliably perform spirometry as per American Thoracic Society/European Respiratory Society criteria [19]. For comparisons of body mass index (BMI) across paediatric and adult age ranges, BMI was stratified into poor, acceptable and well-nourished categories based on percentiles in children ( $\leqslant 5 \%$ ile, $6-50 \%$ ile and $>50 \%$ ile) and absolute BMI in adults [20].

\section{Bacterial isolation and identification}

Standardised culture protocols were used across the three sites as previously described [6], with further details provided in the supplementary material. Briefly, samples were treated for $15 \mathrm{~min}$ with dithiothreitol prior to serial dilution and plating on both selective and nonselective media (supplementary table S1). Plates were incubated under aerobic-micro-aerophilic and anaerobic conditions for between 2 and 7 days. All bacteria detected were quantified $\left(\mathrm{CFU} \cdot \mathrm{g}^{-1}\right.$ or $\mathrm{CFU} \cdot \mathrm{mL}^{-1}$ in $\mathrm{BAL}$ ) by total viable count. Each distinct colonial morphotype was identified by full-length $16 \mathrm{~S}$ rRNA gene sequencing. Strict anaerobes were defined as bacteria known not to survive under atmospheric oxygen tension, whereas facultative anaerobic and aerobic bacteria that grew under anaerobic conditions were included as aerobes in all analyses.

\section{Statistical analysis}

Demographics, markers of disease severity and bacterial prevalence (presence of bacteria at any CFU >0) were compared across clinical sites using the Chi-squared or Fisher's exact test for categorical variables and the Mantel-Haenszel mean score Chi-squared test for ordinal variables. Associations between presence of bacteria and markers of disease severity were assessed by the Mantel-Haenszel mean score Chi-squared test. Analyses were stratified by site as described.

Multivariate logistic regression was used to test for factors that predict prevalence of specific bacterial genera. Predictors were identified via a stepwise model selection (with significance level for entry or staying in the model of $\mathrm{p}=0.10$ or $\mathrm{p}=0.05$, respectively).

Analyses that included bacterial quantities/density were log-transformed to account for nonnormal distributions using $\log _{10}\left(\mathrm{CFU} \cdot \mathrm{g}^{-1}+1\right)$ to incorporate samples with no detectable bacteria. Bacterial diversity measures included richness (number of counted taxa), evenness and diversity (Shannon-Wiener index). Analyses of bacterial communities (network analyses and factor analysis) were conducted on sputum bacterial quantity for the most common genera. Co-occurrence (network analysis) between taxonomic groups was calculated as previously described [21]. Factor analysis as an unsupervised method to group genera was conducted using varimax rotations to achieve independent factor groupings. These factors were used to assign each subject to a group defined by their dominating factor based on similarity of bacterial quantities (see details in supplementary material).

\section{Results}

Subjects and samples

255 subjects were enrolled. Sputum samples $(n=200)$ were obtained at all three sites, whereas BAL $(n=55)$ samples were collected only at UNC $(n=24)$ and Dublin $(n=31)$. All samples from children aged $<6$ years were BAL $(n=39)$. Either sputum $(n=34)$ or BAL $(n=12)$ were collected from subjects aged $6-<18$ years; for subjects aged $\geqslant 18$ years, 166 sputum and four BAL samples were collected.

Subject characteristics by site are shown in table 1. Site-specific differences in the study subjects included older age and fewer subjects positive for F508del mutations in Belfast compared with UNC or Dublin. Fewer subjects in Belfast were undernourished, and, despite being an older cohort, FEV1 values in Belfast were similar to those in UNC and Dublin, consistent with milder disease in Belfast. Use of chronic medications was lower in those contributing BAL versus sputum (supplementary table S2a and b). 
Prevalence and abundance of genera with age

18 anaerobic and 39 aerobic genera were cultured (supplementary table S3), representing 167 species (supplementary table S4). The prevalence of one or more strict anaerobic genus at any CFU $>0$ in a sample was $59 \%$ and was higher in sputum $(67 \%)$ than BAL samples $(31 \%)(\mathrm{p}<0.001)$. Aerobic bacteria were present at any CFU $>0$ in 197 out of 200 (99\%) of sputum and in 51 out of 55 (93\%) BAL samples. The mean \pm SEM total viable counts were higher for aerobic than anaerobic bacteria in sputum $\left(2.27 \times 10^{8} \pm 3.80 \times 10^{7}\right.$ versus $\left.2.86 \times 10^{7} \pm 2.07 \times 10^{7} \mathrm{CFU} \cdot \mathrm{g}^{-1} ; \mathrm{p}<0.001\right)$ and $\mathrm{BAL}\left(3.97 \times 10^{7} \pm 3.71 \times 10^{7}\right.$ versus $\left.2.27 \times 10^{4} \pm 1.61 \times 10^{4} \mathrm{CFU} \cdot \mathrm{mL}^{-1} ; \mathrm{p}<0.001\right)$.

\begin{tabular}{|c|c|c|c|c|c|}
\hline & \multirow[t]{2}{*}{ Overall } & \multicolumn{3}{|c|}{ Clinical site } & \multirow[t]{2}{*}{ p-value } \\
\hline & & Belfast & Chapel Hill & Dublin & \\
\hline Patients & 255 & 75 & 65 & 115 & \\
\hline \multicolumn{6}{|l|}{ Age years } \\
\hline Mean \pm SD & $21.9 \pm 12.7$ & $29.1 \pm 12.2$ & $16.1 \pm 10.3$ & $20.5 \pm 12.1$ & \\
\hline Median (range) & $21.6(1.0-68.2)$ & $26.2(8.3-68.2)$ & $14.5(1.0-50.0)$ & $22.0(1.0-61.2)$ & \\
\hline Age group years & 255 & 75 & 65 & 115 & $<0.0001$ \\
\hline $0-<6$ & $39(15)$ & $0(0)$ & $14(22)$ & $25(22)$ & \\
\hline $6-<13$ & $21(8)$ & $4(5)$ & $13(20)$ & $4(3)$ & \\
\hline $13-<18$ & $25(10)$ & $6(8)$ & $9(14)$ & $10(9)$ & \\
\hline $18-<25$ & $77(30)$ & $22(29)$ & $18(28)$ & 37 (32) & \\
\hline $25-<30$ & $44(17)$ & $19(25)$ & $5(8)$ & $20(17)$ & \\
\hline$\geqslant 30$ & $49(19)$ & 24 (32) & $6(9)$ & $19(17)$ & \\
\hline Sex & 255 & 75 & 65 & 115 & 0.13 \\
\hline Female & 115 (45) & 29 (39) & $36(55)$ & $50(43)$ & \\
\hline Male & $140(55)$ & $46(61)$ & $29(45)$ & $65(57)$ & \\
\hline F508del mutation & 251 & 74 & 63 & 114 & 0.001 \\
\hline Homozygote & $131(52)$ & 29 (39) & $45(71)$ & $57(50)$ & \\
\hline Heterozygote & 99 (39) & $34(46)$ & $15(24)$ & $50(44)$ & \\
\hline None & $21(8)$ & $11(15)$ & $3(5)$ & $7(6)$ & \\
\hline Pancreatic status & 255 & 75 & 65 & 115 & 0.61 \\
\hline Pancreatic sufficiency & 223 (87) & $64(85)$ & $59(91)$ & $100(87)$ & \\
\hline Pancreatic insufficiency & $32(13)$ & $11(15)$ & $6(9)$ & $15(13)$ & \\
\hline BMI & 235 & 74 & 65 & 96 & 0.01 \\
\hline Undernourished & $26(11)$ & $3(4)$ & $9(14)$ & $14(15)$ & \\
\hline Acceptable & $118(50)$ & $34(46)$ & $37(57)$ & 47 (49) & \\
\hline Well nourished & 91 (39) & $37(50)$ & $19(29)$ & $35(36)$ & \\
\hline FEV1 \% pred & 212 & 74 & 51 & 87 & 0.02 \\
\hline$<41$ & $42(20)$ & $10(14)$ & $5(10)$ & $27(31)$ & \\
\hline $41-80$ & $115(54)$ & $48(65)$ & $28(55)$ & $39(45)$ & \\
\hline$>80$ & $55(26)$ & $16(22)$ & $18(35)$ & $21(24)$ & \\
\hline Sample type & 255 & 75 & 65 & 115 & $<0.0001$ \\
\hline BAL & $55(22)$ & $0(0)$ & $24(37)$ & $31(27)$ & \\
\hline Sputum & $200(78)$ & 75 (100) & $41(63)$ & $84(73)$ & \\
\hline Chronic antibiotics & $173(68)$ & $58(77)$ & $43(66)$ & $72(63)$ & 0.10 \\
\hline Flucloxacillin & $18(7)$ & $8(11)$ & $1(2)$ & $9(8)$ & 0.09 \\
\hline Azithromycin & $127(50)$ & 44 (59) & $37(57)$ & $46(40)$ & 0.02 \\
\hline Inhaled antibiotic ${ }^{+}$ & $130(52)$ & $42(56)$ & $30(47)$ & $58(52)$ & 0.58 \\
\hline Any mucolytic & $205(80)$ & $59(79)$ & $54(83)$ & $92(80)$ & 0.79 \\
\hline DNase & $164(64)$ & 54 (72) & $43(66)$ & $67(58)$ & 0.15 \\
\hline Hypertonic saline & $109(43)$ & $15(20)$ & $37(57)$ & $57(50)$ & $<0.0001$ \\
\hline Inhaled corticosteroid & $105(41)$ & $31(41)$ & $36(55)$ & 38 (33) & 0.004 \\
\hline Antacid $^{\S}$ & $129(51)$ & $32(43)$ & $41(63)$ & $56(49)$ & 0.05 \\
\hline Insulin & $34(13)$ & $7(9)$ & $8(12)$ & $19(17)$ & 0.35 \\
\hline
\end{tabular}

Data are presented as $\mathrm{n}$ or $\mathrm{n}(\%)$, unless otherwise stated. BMI: body mass index; FEV1: forced expiratory volume in $1 \mathrm{~s}$; BAL: bronchoalveolar lavage. Patients were continuously enrolled at each site, not necessarily reflecting the study site population. \#: comparisons across sites using the Chi-squared, Mantel-Haenszel mean score Chi-squared or Fisher's exact test; ": based on Global Lung Initiative reference values (see supplementary material); ${ }^{+}$: tobramycin, colistin and aztreonam; $\S_{\text {: }}$ antacids, $\mathrm{H}_{2}$-blockers and proton pump inhibitors. 
The ratio of anaerobic to aerobic viable counts for BAL and sputum showed no differences across age groups (supplementary figure S1a). Bacterial richness was lower in BAL $(3.03 \pm 0.31)$ compared with sputum $(5.18 \pm 0.16)(\mathrm{p}<0.001)$, but similar within each sample type over age sextiles that approximate clinical progression (figure 1a). Shannon diversities showed similar trends (supplementary figure S1b). 16 genera that had $\mathrm{a} \geqslant 5 \%$ prevalence across $\mathrm{BAL}$ and sputum samples are shown by age sextiles in supplementary table S5. The most prevalent anaerobic and aerobic genera across all ages were Prevotella

a)

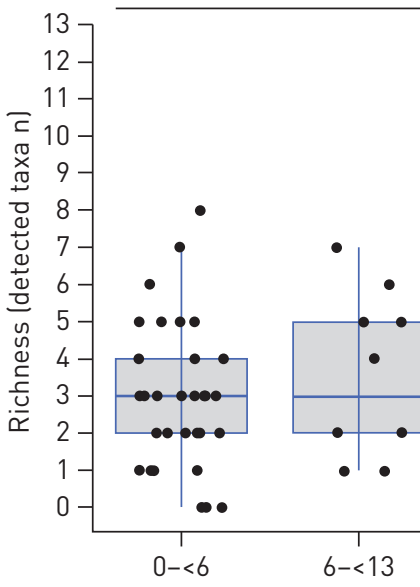

BAL

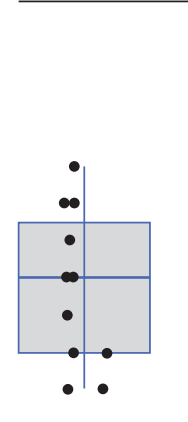

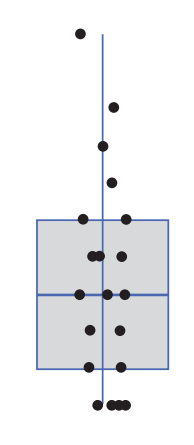

Sputum

b)

$$
\text { }
$$

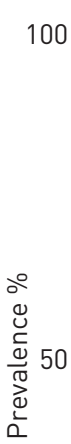

BAL
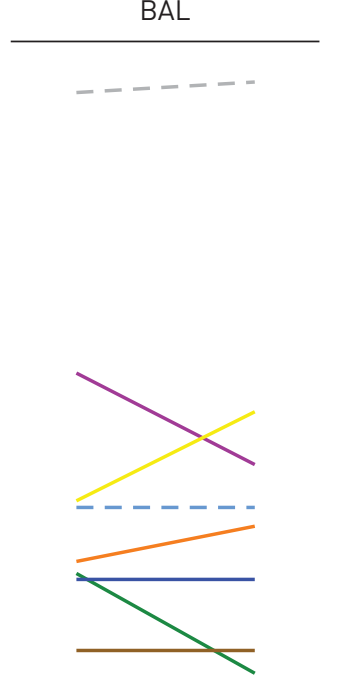

0
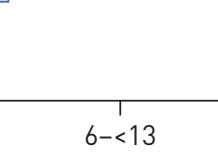

Age range years

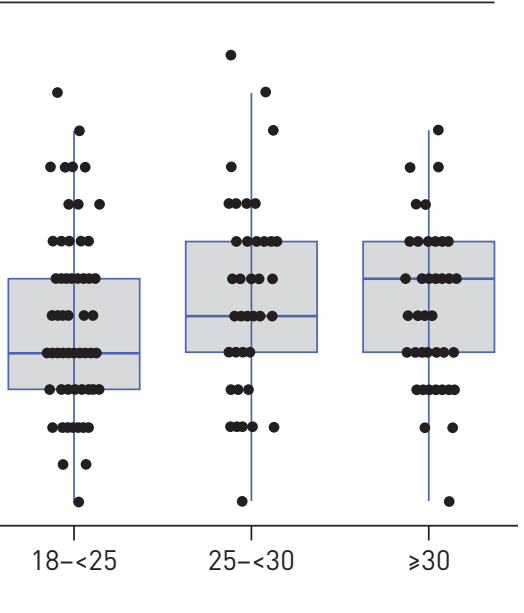


(51\%) and Streptococcus (82\%), respectively. More prevalent bacteria were more abundant with the exception of Burkholderia with a 5\% prevalence, but being most abundant when present, and Prevotella with high prevalence, but low abundance. Changes in prevalence of the most frequently cultured anaerobes and aerobes with age showed decreasing prevalence from childhood to mid-adulthood, with subsequent increases for anaerobic genera and Streptococcus. Inverse trends were seen for Pseudomonas (figure 1b).

Analyses of samples by sites revealed differences in bacterial richness across sites, but similar diversity in sputum samples (supplementary figure S2a). There were no differences in richness or diversity in BAL samples from Dublin versus UNC (supplementary figure S2b). The prevalence of Pseudomonas was highest at UNC, with Burkholderia, Haemophilus, Actinomyces, Rothia and Gemella being more prevalent in Belfast. Neisseria prevalence was lowest in Belfast. Dublin had the lowest prevalence of Prevotella and Veillonella. Further details are shown by site and sample type in supplementary table S3 and supplementary figure S3.

\section{Relationship between bacterial prevalence, abundance and disease severity}

In subjects who produced sputum $(\mathrm{n}=200)$, anaerobes were associated with phenotypically milder disease, e.g. better lung function and BMI, and with pancreatic sufficiency, absence of insulin and chronic
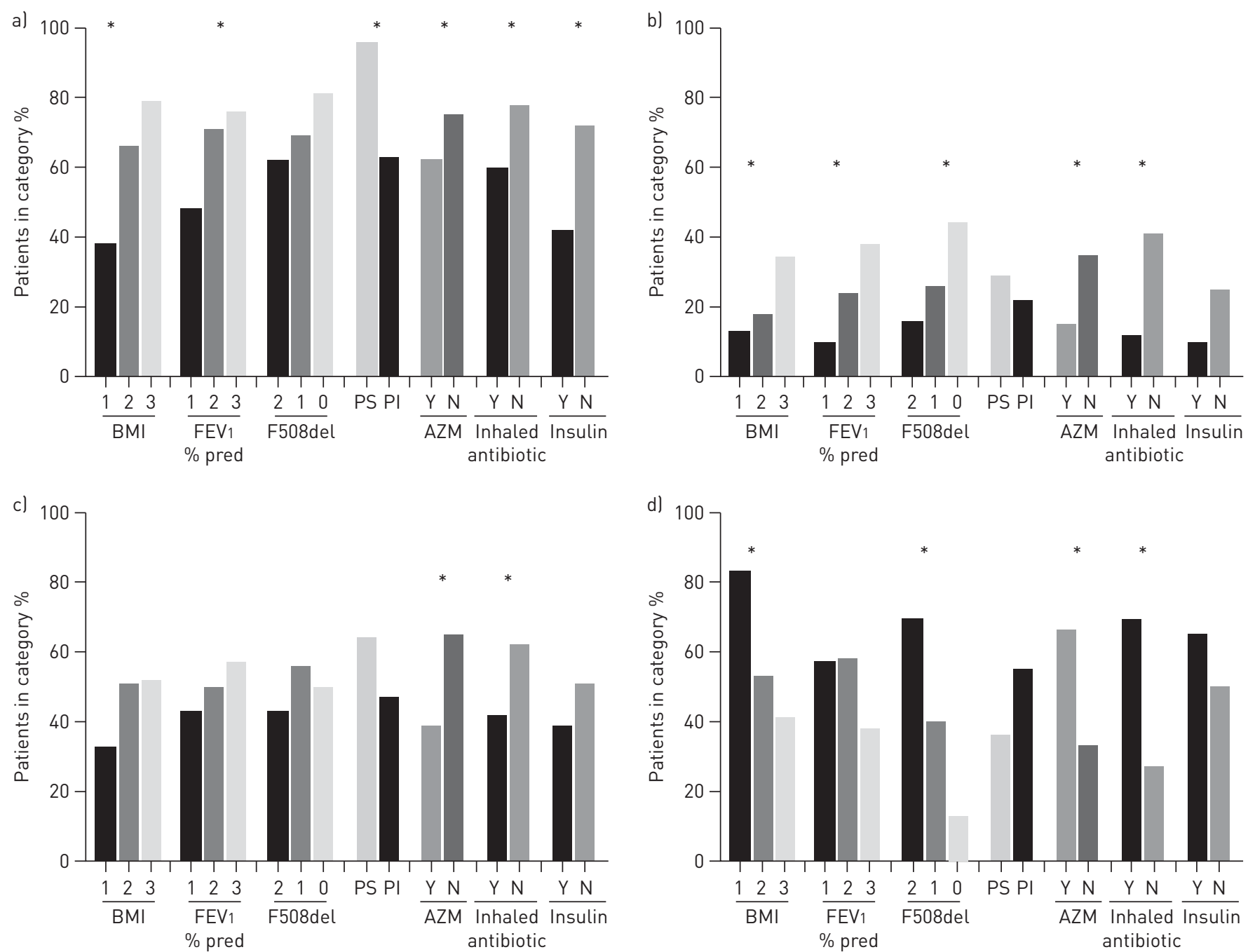

FIGURE 2 Prevalence as percentage of subjects within the category with any CFU $>0$ for a) anaerobes, b) Haemophilus, c) Staphylococcus and d) Pseudomonas in sputum and their association with clinical characteristics. BMI: body mass index; FEV1: forced expiratory volume in $1 \mathrm{~s}$; PS: pancreatic sufficiency; PI: pancreatic insufficiency; AZM: azithromycin. 200 sputum samples included. Seven subjects had missing data for FEV 1 and eight subjects had missing data for BMI. BMI categories are 1: poor nutritional status; 2: adequate; 3: well nourished. FEV1 \% pred categories are 1: $<40 \% ; 2: 40-80 \% ; 3:>80 \%$ (see supplementary material). Numbers per category are given as percentage of subjects positive for this characteristic, where each category (e.g. "BMI 1") can reach 100\%. Inhaled antibiotics included tobramycin, colistin and aztreonam. *: significant difference between groups by the Mantel-Haenszel mean score Chi-squared test stratified by site. 


\begin{tabular}{|c|c|c|c|c|}
\hline & Anaerobes & Haemophilus & Staphylococcus & Pseudomonas \\
\hline Patients n & 197 & 197 & 200 & 195 \\
\hline F508del 1 versus 0 & & & & $8.24(1.00-68.20)$ \\
\hline F508del 2 versus 0 & & & & $17.63(2.13-146.04)$ \\
\hline Pancreatic insufficiency & $0.12(0.03-0.53)$ & & & \\
\hline Azithromycin & & $0.41(0.18-0.92)$ & $0.35(0.19-0.62)$ & $2.26(1.10-4.67)$ \\
\hline Inhaled antibiotic & & $0.26(0.12-0.57)$ & & $3.97(1.96-8.05)$ \\
\hline Centre C versus B & $0.58(0.22-1.52)^{\#}$ & $0.41(0.15-1.08)^{\#}$ & & \\
\hline Centre D versus B & $0.17(0.08-0.38)$ & $0.17(0.07-0.41)$ & & \\
\hline
\end{tabular}

Data are presented as OR (95\% CI), unless otherwise stated. Variables evaluated for model inclusion were: sex, age, centre (B: Belfast; C: Chapel Hill; D: Dublin), forced expiratory volume in $1 \mathrm{~s}$ and body mass index as categories (as in figure 2), F508del, pancreatic status, chronic use of flucloxacillin, azithromycin, inhaled antibiotics, DNase, hypertonic saline, corticosteroids, and antacids. Blank cells indicate these variables were not selected for the final model because they were not significant predictors as determined by stepwise model selection with $p=0.10$ for entry and $p=0.05$ for staying in the model. \#: difference is not significant but comparison included in model for comparison to centre D versus B.

antibiotic use, but not genotype (figure 2a). Haemophilus influenzae, an aerobe observed in early CF disease, followed a similar pattern (figure 2b). Staphylococcus exhibited an intermediate pattern with negative associations, i.e. was less prevalent, with use of chronic antibiotics (figure 2c). The pattern for Pseudomonas, however, was distinctly different from anaerobes. Pseudomonas prevalence was positively associated with worse BMI, number of F508del alleles and chronic antibiotics (figure 2d).

Logistic regression adjusting for covariates with $\mathrm{p}<0.05$ identified through stepwise modelling revealed a lower prevalence of anaerobes in pancreatic-insufficient subjects (table 2). With respect to aerobes, a lower prevalence of Haemophilus and Staphylococcus was associated with use of azithromycin. Pseudomonas prevalence again was associated with F508del alleles, and both azithromycin and inhaled antibiotic use. For BAL, clinical or site variables were not associated with bacterial prevalence.

No associations were observed for absolute anaerobic or aerobic viable counts and clinical variables or study site in sputum or BAL. However, calculation of the anaerobe/aerobe CFU ratio showed that subjects with a relatively higher load of anaerobes had milder disease (pancreatic sufficiency and higher BMI) than aerobe-dominated subjects (supplementary figure S4).

\section{Community structure in sputum and association with subject characteristics}

Bacterial communities reflecting the 16 genera with a $\geqslant 5 \%$ prevalence were explored in sputum samples $(\mathrm{n}=200)$ using microbial co-occurrence network analysis. Bacteria typical of the oropharynx, e.g. Streptococcus, Rothia and Gemella, associated with each other. In contrast, Staphylococcus, Pseudomonas and Burkholderia each formed distinct entities (figure 3).

Next, factor analysis was used to reduce the complexity of the microbiota per sample into a set of variables that best defined sample community composition. The bacterial community compositions were generally consistent with the co-occurrence networks. Five components/factors explained $49 \%$ of the variation (figure 4). Factors 1 and 2 were dominated by oral-associated bacteria, including Streptococcus, Gemella and Rothia. Factor 3 included Haemophilus and Neisseria. Factor 4 was dominated by obligate anaerobes. Pseudomonas defined Factor 5, which was also negatively associated with Staphylococcus and Burkholderia.

To define the clinical relevance of these microbial communities, patient sputum samples were defined by their dominating factor (table 3). Thus, each sample was assigned to Groups 1-5. There were no differences in pancreatic status, use of azithromycin, mucolytics and anaerobic or aerobic bacterial load/ density between groups. Groups 4 and 5 had the highest percentage of F508del homozygous subjects, yet differed significantly with regard to both anaerobes and Pseudomonas. The group with higher anaerobic density/diversity (Group 4) tended to have a higher FEV1 and a lower incidence of poor nutritional status compared with the Pseudomonas-dominated Group 5. Notably, Group 3 included the fewest subjects with poor nutritional status and the most with a high FEV1, compared with other groups, but had the highest proportion of subjects aged $<20$ years.

\section{Discussion}

Anaerobic and aerobic bacteria were cultured from respiratory samples across all age ranges to assess bacterial prevalence and quantify density in CF respiratory secretions. The prevalence and quantities of both 


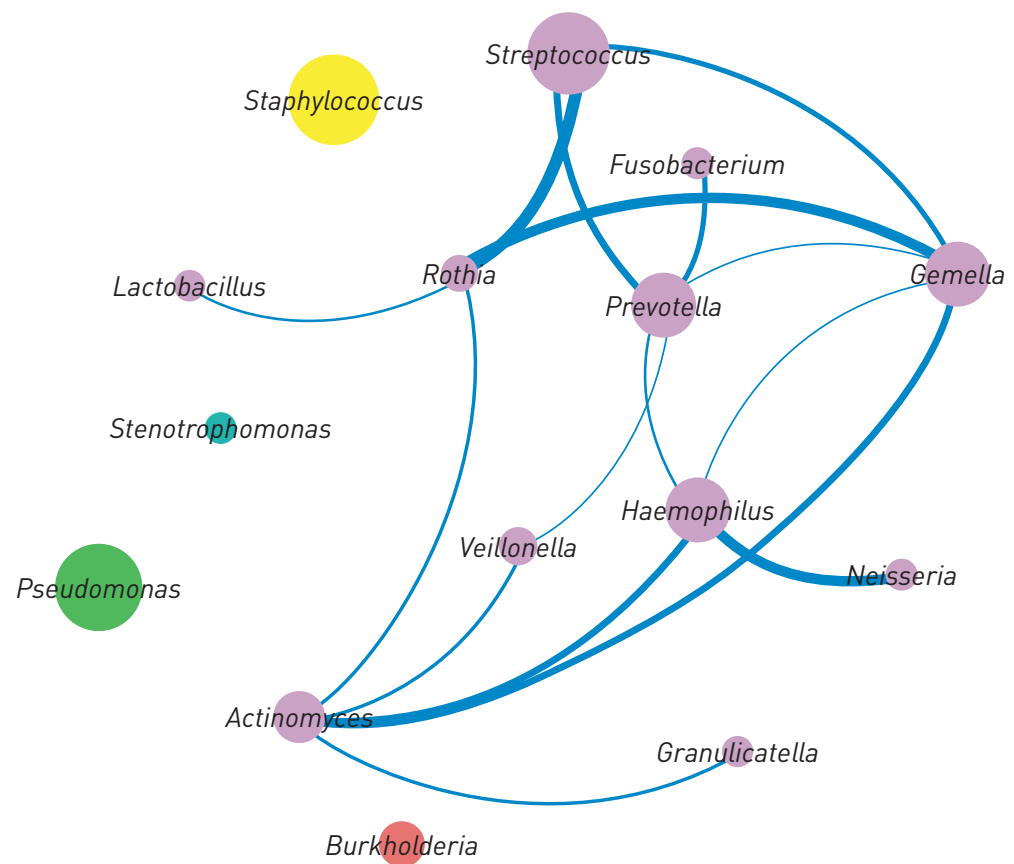

Porphyromonas

FIGURE 3 Bacterial co-occurrence network between genera detected by extended microbial culture. The 16 genera detected at $a \geqslant 5 \%$ prevalence from sputum samples are contained within the network. Co-occurring microbial taxa are shown with nodes (circles) denoting a particular genus within the network and each edge (blue lines) denoting a significant co-occurrence relationship (Spearman's rank correlation coefficient) for positive correlations lines); adjusted p-value $<0.05$ (Benjamini-Hochberg-Yekutieli false discovery rate correction; see supplementary material). The size of the corresponding nodes demonstrates the relative proportion of each genus within the current study and the thickness of the edges represents the strength of the corresponding associations.

anaerobic and aerobic bacteria were lower in BAL compared with sputum. Interestingly, the anaerobe/aerobe ratios in BAL, i.e. incorporating quantity, were similar to sputum. Facultative anaerobes and micro-aerophilic bacteria were also cultured in both sample types (supplementary table S3) and were included with aerobes, potentially underestimating the true anaerobic burden in the CF lung. Pseudomonas and Staphylococcus also grow anaerobically, a growth mode associated with persistence in chronic infection, but the focus in this study was on strict anaerobes as determined in molecular studies. The prevalence of anaerobes and Streptococcus declined from early childhood to early adulthood, i.e. a time when many patients experience worsening disease. In older age groups, anaerobes were more prevalent again, possibly related to less severe disease (figure $1 \mathrm{~b}$ ) as reflected by the higher anaerobe/aerobe ratio in subjects with better nutritional status (supplementary figure S4). Anaerobic and streptococcal species are highly abundant in oral secretions, and are detected in the oral cavity in the first months of life [22]. Although oral secretions are a source of contamination of sputum and BAL, the high abundance of anaerobic genera makes sample contamination an unlikely explanation for our findings. Although BAL samples were obtained via a laryngeal mask airway or endotracheal tube, which decreases the risk of contamination, the lower anaerobe prevalence in BAL compared with sputum likely reflects the younger BAL subject age with lower bacterial load and sample dilution. Aerobe prevalence and abundance were also lower in BAL than sputum. Typically, BAL is assumed to represent a 30 - to 80 -fold dilution of epithelial lining fluid $[23,24]$.

The source of CF lower airways oral bacteria likely reflects micro-aspiration as reported in healthy subjects, in whom oral bacteria are found in proximal airways but continuous mucociliary clearance prevents growth to high densities $[25,26]$. We suggest that starting in infancy, abnormal mucociliary clearance in $\mathrm{CF}$ enhances retention of aspirated flora in an anaerobic airways environment, allowing anaerobic bacteria to proliferate. Such a scenario is consistent with recent CF infant BAL microbiome analyses [27-29].

The contribution of anaerobic bacteria to lung disease is not clearly understood. This large study, which analysed samples from $255 \mathrm{CF}$ subjects across three sites and a wide age range, characterised the anaerobic and aerobic microbial community composition in the CF airways using extended culture methods. A prior molecular study had shown geographical differences in the CF microbiome [30]. In our study, differences occurred by study site but not by continent, making climate a less relevant factor than genotype (e.g. F508del) and associated disease severity. We observed that anaerobic bacteria were more prevalent in 


\begin{tabular}{|c|c|c|c|c|c|}
\hline & Factor 1 & Factor 2 & Factor 3 & Factor 4 & Factor 5 \\
\hline Streptococcus & 0.672 & -0.056 & 0.166 & -0.032 & -0.076 \\
\hline Gemella & 0.574 & 0.274 & 0.087 & 0.109 & -0.098 \\
\hline Rothia & 0.550 & 0.434 & -0.162 & 0.121 & 0.004 \\
\hline Stenotrophomonas & 0.496 & -0.329 & -0.123 & -0.138 & 0.300 \\
\hline Granulicatella & 0.035 & 0.608 & 0.219 & -0.095 & 0.150 \\
\hline Lactobacillus & -0.085 & 0.597 & -0.282 & 0.122 & -0.081 \\
\hline Actinomyces & 0.320 & 0.562 & 0.135 & -0.094 & -0.235 \\
\hline Neisseria & 0.028 & -0.075 & 0.736 & -0.057 & 0.106 \\
\hline Haemophilus & 0.162 & 0.263 & 0.654 & 0.123 & -0.166 \\
\hline Fusobacterium & -0.182 & 0.101 & 0.049 & 0.654 & 0.209 \\
\hline Porphyromonas & 0.014 & -0.201 & -0.162 & 0.630 & 0.022 \\
\hline Prevotella & 0.378 & -0.027 & 0.352 & 0.557 & -0.092 \\
\hline Veillonella & 0.165 & 0.275 & 0.108 & 0.454 & -0.236 \\
\hline Pseudomonas & -0.034 & -0.097 & -0.051 & 0.051 & 0.735 \\
\hline Staphylococcus & -0.245 & -0.265 & 0.325 & 0.160 & -0.491 \\
\hline Burkholderia & 0.179 & 0.039 & -0.318 & -0.097 & -0.533 \\
\hline Eigenvalue & 2.42 & 1.62 & 1.37 & 1.31 & 1.16 \\
\hline Variance explained \% & 11.41 & 9.94 & 9.80 & 9.31 & 8.90 \\
\hline
\end{tabular}

FIGURE 4 Loadings from factor analysis showing groupings of bacteria with each other based on quantity in sputum ( $n=200$ samples). Five factors explain $49 \%$ of the total variation. Blue indicates positive associations and red indicates negative associations, with darker colours indicating the robustness of associations.

sputum of subjects with milder disease. Higher lung function, increased BMI, pancreatic sufficiency and absence of requirement for insulin were associated with higher prevalence of anaerobes in univariate analyses (figure 2). Lower prevalence of anaerobes and Haemophilus in subjects on azithromycin could be due to the antibacterial activity of azithromycin. Azithromycin exhibits good activity against those organisms in non-CF disease; however, high rates of resistance are seen in CF that may mitigate its activity $[31,32]$. Notably, associations with pancreatic sufficiency remained significant when adjusting for multiple medications and clinical parameters (table 2). A sensitivity analyses with $\mathrm{p}=0.1$ for staying in the model additionally included $\mathrm{FEV}_{1}$ for Pseudomonas, azithromycin and insulin for anaerobes, and hypertonic saline for Haemophilus (supplementary table S6).

Our culture-based findings are consistent with observations from molecular microbiome studies describing higher diversity with less severe CF lung disease [5,33] and decreasing diversity in patients with more rapid lung function decline $[5,11]$. Our quantitative cultures allowed these relationships to be extended to anaerobic and aerobic bacterial density, anaerobe/aerobe ratio and diversity. A higher anaerobe/aerobe ratio was associated with pancreatic sufficiency and better nutrition, with trends for higher lung function in those with a higher anaerobe/aerobe ratio (supplementary figure S4). However, associations between absolute aerobic or anaerobic bacterial concentrations/density and FEV1 were not detected. We hypothesise that the absence of such an association partly reflects the spatial heterogeneity of CF lung disease, with bacterial density reflecting disease severity in focal bronchiectatic areas, whereas FEV1 reflects the overall extent of diseased lung. The hypothesis that FEV1 is not sufficiently sensitive to detect regional, bronchiectatic disease is supported by a study that reported associations between anaerobic density in CF patients and lung clearance index, but not FEV1 [34]. Moreover, it should be noted that age-based CF-specific lung function parameters developed for children [35] are not available for adults and effects of genetic mutations on lung function were not included. Thus, although we used Global Lung Initiative reference equations (see supplementary material) for comparison across body stature and centre, analyses of relationships of bacterial density with FEV1 may have been more sensitive if CF- and age-specific references were available.

Analyses of anaerobe pathogenicity in vitro may also yield insight into the relationships between anaerobes and CF disease severity. Prevotella was the most abundant and prevalent anaerobic genus in this study, 
TABLE 3 Patient characteristics within the groups characterised by bacterial communities identified by factor analysis

\begin{tabular}{|c|c|c|c|c|c|c|}
\hline & Group 1 & Group 2 & Group 3 & Group 4 & Group 5 & p-value ${ }^{\#}$ \\
\hline Age years & $27.56 \pm 1.49$ & $28.62 \pm 1.75$ & $22.99 \pm 1.53$ & $24.34 \pm 1.99$ & $26.82 \pm 1.56$ & 0.089 \\
\hline Homozygote F508del & 40 & 54 & 37 & 64 & 61 & 0.046 \\
\hline Null F508del & 15 & 11 & 6 & 4 & 2 & 0.2 \\
\hline FEV $1 \%$ pred & $59.47 \pm 3.17^{f}$ & $55.79 \pm 3.78^{+}$ & $75.64 \pm 3.28^{+, \S . f}$ & $61.58 \pm 4.26$ & $57.08 \pm 3.35^{\S}$ & 0.0002 \\
\hline Inhaled antibiotic use & 67 & 66 & 35 & 56 & 78 & 0.0007 \\
\hline Pseudomonas-positive & 38 & 37 & 39 & 59 & 91 & $<0.0001$ \\
\hline Staphylococcus-positive & 31 & 49 & 78 & 70 & 27 & $<0.0001$ \\
\hline Any anaerobe & 81 & 60 & 72 & 100 & 32 & $<0.0001$ \\
\hline Aerobe richness & $6.83 \pm 0.43^{+}$ & $6.69 \pm 0.50^{\S}$ & $6.07 \pm 0.44$ & $5.7 \pm 0.57^{\Uparrow}$ & $4.57 \pm 0.45^{+, \S}$ & 0.0038 \\
\hline Anaerobe richness & $1.75 \pm 1.19^{+}$ & $1.20 \pm 0.23$ & $1.59 \pm 0.20^{\S}$ & $3.22 \pm 0.26^{\pi}$ & $0.68 \pm 0.21^{+, \S}$ & $<0.0001$ \\
\hline
\end{tabular}

Data are presented as mean \pm SD or $\%$, unless otherwise stated. BMI: body mass index; FEV1: forced expiratory volume in $1 \mathrm{~s}$. Groups 1 and 2 dominated by oral bacteria; Group 3 Haemophilus and Neisseria; Group 4 anaerobes; Group 5 Pseudomonas-dominated. Inhaled antibiotics included tobramycin, colistin and aztreonam. " : p-values for comparison of categorical (Pearson) or continuous variables (ANOVA) across groups ( $<<0.05$ considered significant). Adjusted difference (Tukey's): ๆ: differs from all other groups; ${ }^{+}$(.f. : differences to corresponding symbol by row.

and has been reported consistently in other studies [5, 6, 10, 36-38]. In vitro studies of laboratory strains of Prevotella demonstrated that they produce short-chain fatty acids and proteases that may be pro-inflammatory [9]. However, Prevotella elicits diminished pro-inflammatory responses in respiratory cell lines and murine models compared with classic aerobic pathogens [39]. Thus, conceivably the relatively lower virulence of anaerobes contributes to their association with a milder CF phenotype.

Co-occurrence networks to visualise potential bacterial interactions showed groupings of orally derived anaerobic bacteria, which occupied niches distinct from the known pathogens (e.g. Pseudomonas and Staphylococcus) that were themselves highly dissimilar (figure 3). Both Burkholderia and Pseudomonas release products that are toxic to other bacteria, which may contribute to their distinct niches as well as their dominance [40]. CF registry data also demonstrate decreasing prevalence of Staphylococcus in older patients as Pseudomonas prevalence increases, suggesting a similar community competition for these two bacteria $[41,42]$.

Factor analyses (figure 4) revealed bacterial groupings similar to the co-occurrence networks. These factors enabled testing of associations with clinical parameters based on samples defined by the highest factor loadings (table 3). Group 4 with the highest prevalence and diversity of anaerobes had milder disease than the Pseudomonas-dominated group, but not the mildest disease, possibly reflecting that Group 4 exhibited the highest proportion of F508del homozygote subjects and moderate/high rates of Pseudomonas.

A challenge to the present study is the inclusion of paediatric and adult age groups, necessitating comparisons between BAL and sputum. For age-wide comparisons, prevalence was used as a surrogate in addition to the ratio of anaerobic to aerobic bacterial load. Similar to prior studies, this study is limited by its observational design and thus cannot determine the association versus causality with respect to anaerobe-induced disease severity. This difference has an impact when considering targeted anaerobe antibiotic therapy in subjects with CF. A recent study in infants with CF demonstrated more airway inflammation in children whose airway microbiome was dominated by anaerobes compared with "sterile" airways [29]. Anaerobes may also condition the lower CF airways to promote infection with more classic pathogens [43]. Such data may support treatment of anaerobes in early CF disease. In contrast, bacterial culture and molecular studies in older, sputum-producing subjects suggest that a diverse lung microbiota with relatively abundant anaerobes is associated with milder disease. Conceivably, higher anaerobic diversity in adults with milder disease reflects lower antibiotic use in such subjects [44] and/or lower intrinsic virulence of anaerobes [39]. Therefore, targeted "eradication" of anaerobes in older patients, when clinically at baseline, might result in little clinical benefit and more resistant organisms. Anaerobes may increase at onset of exacerbations and therefore their role may fluctuate with short-term changes in disease state $[6,14]$. Anaerobes are not routinely cultured for in the clinical setting. This approach may be justified in older subjects if antimicrobial therapy is not being considered. 
In summary, anaerobic bacteria were detected in CF respiratory secretions at all ages. In the age groups investigated in this study, aerobes dominated over anaerobes with respect to bacterial numbers. The prevalence of anaerobic genera was associated with milder disease and lower use of antibiotics, suggesting that a diverse anaerobic microbiota may be a marker of better health in older subjects with CF. Sputum anaerobic/aerobic bacterial genera clustered into communities, which were associated with clinical outcomes. Cross-sectional studies do not permit the distinction of anaerobes as markers versus causative factors in the severity of lung disease. Collectively, our data suggest that targeted eradication of anaerobes in the context of aerobic pathogenic bacteria may not be warranted until further data are available.

Acknowledgements: We would like to thank the patients and families who participated in this study, and the clinical teams at each site who facilitated completion of the study. We acknowledge all staff involved in laboratory culture of bacteria and patient recruitment.

Conflict of interest: G.G. Einarsson's work is supported by a grant from IMI (iABC; Inhaled Antibiotics in Bronchiectasis and Cystic Fibrosis). N. Gotman reports grants from AECF, during the conduct of the study. S. Davis Thomas reports grants from the National Institutes of Health $(\mathrm{NIH})$, during the conduct of the study. P.H. Gilligan reports grants from the NIH, during the conduct of the study. M.C. Wolfgang reports grants from the NIH, during the conduct of the study. J.S. Elborn reports grants from Northern Ireland Research and Development, during the conduct of the study. R.C. Boucher is Chairman of the Board of Parion Sciences, a privately held UNC spin-out company focused on developing therapies for $\mathrm{CF}$, and has received monetary compensation for this role. M.M. Tunney reports grants from Health and Social Care Research and Development Division, Public Health Agency, Northern Ireland, during the conduct of the study; and grants from Alaxia, outside the submitted work.

Support statement: Work at UNC was supported by the National Institutes of Health (NIH) under grants HL084934, HL100809, P30DK065988 and P0HL108808. Research at Queen's University Belfast was funded by the Health and Social Care Research and Development Division, Public Health Agency, Northern Ireland and the Medical Research Council through a US-Ireland Partnership Grant. M.M. Tunney was supported by a Health and Social Care Research and Development, Public Health Agency, Northern Ireland-funded UK National Institute for Health Research Career Scientist Award. Research at Royal College of Surgeons in Ireland, Dublin was supported by the Science Foundation Ireland and the Health Research Board under grant SFI/08/US/B1676 and by the NIH under grant 5R01 HL092964-04. Funding information for this article has been deposited with the Crossref Funder Registry.

\section{References}

1 Sherrard LJ, Tunney MM, Elborn JS. Antimicrobial resistance in the respiratory microbiota of people with cystic fibrosis. Lancet 2014; 384: 703-713.

2 Worlitzsch D, Tarran R, Ulrich M, et al. Effects of reduced mucus oxygen concentration in airway Pseudomonas infections of cystic fibrosis patients. J Clin Invest 2002; 109: 317-325.

3 Cowley ES, Kopf SH, LaRiviere A, et al. Pediatric cystic fibrosis sputum can be chemically dynamic, anoxic, and extremely reduced due to hydrogen sulfide formation. MBio 2015; 6: e00767.

4 Worlitzsch D, Rintelen C, Bohm K, et al. Antibiotic-resistant obligate anaerobes during exacerbations of cystic fibrosis patients. Clin Microbiol Infect 2009; 15: 454-460.

5 Coburn B, Wang PW, Diaz Caballero J, et al. Lung microbiota across age and disease stage in cystic fibrosis. Sci Rep 2015; 5: 10241.

6 Tunney MM, Field TR, Moriarty TF, et al. Detection of anaerobic bacteria in high numbers in sputum from patients with cystic fibrosis. Am J Respir Crit Care Med 2008; 177: 995-1001.

7 Brown PS, Pope CE, Marsh RL, et al. Directly sampling the lung of a young child with cystic fibrosis reveals diverse microbiota. Ann Am Thorac Soc 2014; 11: 1049-1055.

8 Ghorbani P, Santhakumar P, Hu Q, et al. Short-chain fatty acids affect cystic fibrosis airway inflammation and bacterial growth. Eur Respir J 2015; 46: 1033-1045.

9 Mirkovic B, Murray MA, Lavelle GM, et al. The role of short-chain fatty acids, produced by anaerobic bacteria, in the cystic fibrosis airway. Am J Respir Crit Care Med 2015; 192: 1314-1324.

10 Cox MJ, Allgaier M, Taylor B, et al. Airway microbiota and pathogen abundance in age-stratified cystic fibrosis patients. PLoS One 2010; 5: e11044.

11 Zhao J, Schloss PD, Kalikin LM, et al. Decade-long bacterial community dynamics in cystic fibrosis airways. Proc Natl Acad Sci USA 2012; 109: 5809-5814.

12 Young GR, Smith DL, Embleton ND, et al. Reducing viability bias in analysis of gut microbiota in preterm infants at risk of NEC and sepsis. Front Cell Infect Microbiol 2017; 7: 237.

13 Nguyen LD, Deschaght P, Merlin S, et al. Effects of propidium monoazide (PMA) treatment on mycobiome and bacteriome analysis of cystic fibrosis airways during exacerbation. PLoS One 2016; 11: e0168860.

14 Lim YW, Evangelista JS III, Schmieder R, et al. Clinical insights from metagenomic analysis of sputum samples from patients with cystic fibrosis. J Clin Microbiol 2014; 52: 425-437.

15 Klappenbach JA, Saxman PR, Cole JR, et al. rrndb: the Ribosomal RNA Operon Copy Number Database. Nucleic Acids Res 2001; 29: 181-184.

16 Vetrovsky T, Baldrian P. The variability of the 16S rRNA gene in bacterial genomes and its consequences for bacterial community analyses. PLoS One 2013; 8: e57923.

17 Sherrard LJ, Schaible B, Graham KA, et al. Mechanisms of reduced susceptibility and genotypic prediction of antibiotic resistance in Prevotella isolated from cystic fibrosis (CF) and non-CF patients. J Antimicrob Chemother 2014; 69: 2690-2698.

18 Sherrard LJ, McGrath SJ, McIlreavey L, et al. Production of extended-spectrum beta-lactamases and the potential indirect pathogenic role of Prevotella isolates from the cystic fibrosis respiratory microbiota. Int $J$ Antimicrob Agents 2016; 47: 140-145. 
19 Laszlo G. Standardisation of lung function testing: helpful guidance from the ATS/ERS Task Force. Thorax 2006; 61: 744-746.

20 Turck D, Braegger CP, Colombo C, et al. ESPEN-ESPGHAN-ECFS guidelines on nutrition care for infants, children, and adults with cystic fibrosis. Clin Nutr 2016; 35: 557-577.

21 Einarsson GG, Comer DM, McIlreavey L, et al. Community dynamics and the lower airway microbiota in stable chronic obstructive pulmonary disease, smokers and healthy non-smokers. Thorax 2016; 71: 795-803.

22 Kononen E. Development of oral bacterial flora in young children. Ann Med 2000; 32: 107-112.

23 Singh S, Grover V, Christie L, et al. A comparative study of bronchoscopic microsample probe versus bronchoalveolar lavage in patients with burns-related inhalational injury, acute lung injury and chronic stable lung disease. Respiration 2015; 89: 19-26.

24 Braun J, Mehnert A, Dalhoff $\mathrm{K}$, et al. Different BALF protein composition in normal children and adults. Respiration 1997; 64: 350-357.

25 Dickson RP, Erb-Downward JR, Freeman CM, et al. Spatial variation in the healthy human lung microbiome and the adapted island model of lung biogeography. Ann Am Thorac Soc 2015; 12: 821-830.

26 Bassis CM, Erb-Downward JR, Dickson RP, et al. Analysis of the upper respiratory tract microbiotas as the source of the lung and gastric microbiotas in healthy individuals. MBio 2015; 6: e00037.

27 Subbarao P, Milla C, Aurora P, et al. Multiple-breath washout as a lung function test in cystic fibrosis. A Cystic Fibrosis Foundation Workshop Report. Ann Am Thorac Soc 2015; 12: 932-939.

28 Frayman KB, Armstrong DS, Carzino R, et al. The lower airway microbiota in early cystic fibrosis lung disease: a longitudinal analysis. Thorax 2017; 72: 1104-1112.

29 Muhlebach MS, Zorn BT, Esther CR, et al. Initial acquisition and succession of the cystic fibrosis lung microbiome is associated with disease progression in infants and preschool children. PLoS Pathog 2018; 14: e1006798.

30 Stressmann FA, Rogers GB, Klem ER, et al. Analysis of the bacterial communities present in lungs of patients with cystic fibrosis from American and British centers. J Clin Microbiol 2011; 49: 281-291.

31 Farrell DJ, Flamm RK, Sader HS, et al. Results from the Solithromycin International Surveillance Program (2014). Antimicrob Agents Chemother 2016; 60: 3662-3668.

32 Sherrard LJ, Graham KA, McGrath SJ, et al. Antibiotic resistance in Prevotella species isolated from patients with cystic fibrosis. J Antimicrob Chemother 2013; 68: 2369-2374.

33 Fodor AA, Klem ER, Gilpin DF, et al. The adult cystic fibrosis airway microbiota is stable over time and infection type, and highly resilient to antibiotic treatment of exacerbations. PLoS One 2012; 7: e45001.

34 O'Neill K, Bradley JM, Johnston E, et al. Reduced bacterial colony count of anaerobic bacteria is associated with a worsening in lung clearance index and inflammation in cystic fibrosis. PLoS One 2015; 10: e0126980.

35 Kulich M, Rosenfeld M, Campbell J, et al. Disease-specific reference equations for lung function in patients with cystic fibrosis. Am J Respir Crit Care Med 2005; 172: 885-891.

36 Tunney MM, Klem ER, Fodor AA, et al. Use of culture and molecular analysis to determine the effect of antibiotic treatment on microbial community diversity and abundance during exacerbation in patients with cystic fibrosis. Thorax 2011; 66: 579-584.

37 Zemanick ET, Wagner BD, Robertson CE, et al. Airway microbiota across age and disease spectrum in cystic fibrosis. Eur Respir J 2017; 50: 1700832.

38 Harris JK, De Groote MA, Sagel SD, et al. Molecular identification of bacteria in bronchoalveolar lavage fluid from children with cystic fibrosis. Proc Natl Acad Sci USA 2007; 104: 20529-20533.

39 Larsen JM, Musavian HS, Butt TM, et al. Chronic obstructive pulmonary disease and asthma-associated Proteobacteria, but not commensal Prevotella spp., promote Toll-like receptor 2-independent lung inflammation and pathology. Immunology 2015; 144: 333-342.

40 de la Fuente-Nunez C, Reffuveille F, Fernandez L, et al. Bacterial biofilm development as a multicellular adaptation: antibiotic resistance and new therapeutic strategies. Curr Opin Microbiol 2013; 16: 580-589.

41 Cystic Fibrosis Foundation. Patient Registry Annual Report. 2013. www.cff.org/2013_CFF_Patient_Registry_ Annual_Data_Report.pdf Date last accessed: May 31, 2018.

42 UK Cystic Fibrosis Registry. Annual Report. 2015. www.cysticfibrosis.org.uk/the-work-we-do/uk-cf-registry Date last accessed: January 31, 2018.

43 Flynn JM, Niccum D, Dunitz JM, et al. Evidence and role for bacterial mucin degradation in cystic fibrosis airway disease. PLoS Pathog 2016; 12: e1005846.

44 Zhao J, Murray S, Lipuma JJ. Modeling the impact of antibiotic exposure on human microbiota. Sci Rep 2014; 4: 4345 . 